\title{
Conceptualizing Design Knowledge in IS Research - A Review and Taxonomy of Design Knowledge Properties
}

\author{
Ernestine Dickhaut \\ University of Kassel, Germany \\ ernestine.dickhaut@uni-kassel.de
}

\author{
Andreas Janson \\ University of St.Gallen, Switzerland \\ andreas.janson@unisg.ch
}

\author{
Jan Marco Leimeister \\ University of Kassel, Germany \& University of St.Gallen, Switzerland \\ leimeister@uni-kassel.de
}

\begin{abstract}
Design science projects are of great interest in information systems (IS) research. Typically, designoriented projects generate valuable design knowledge through the design and possible instantiation of artifacts. Although designing novel artifacts and accumulating design knowledge is common practice in $I S$, there is still limited shared knowledge about the distinctive characteristics of design knowledge to facilitate its accumulation. To address this issue, we develop a design knowledge taxonomy and contribute to a deeper understanding of design knowledge properties. The taxonomy is grounded on a systematic literature review, followed by a combination of empirical-to-conceptual and conceptual-to-empirical iterations. We evaluate the taxonomy by interviewing six domain experts and demonstrate its practical application and utility. Thus, the taxonomy consists of key dimensions and characteristics of design knowledge and contributes to a better scientific understanding of its characteristics. Practitioners can use the taxonomy as an instrument to further understand, design, and accumulate design knowledge.
\end{abstract}

\section{Introduction}

Design science research (DSR) projects and the design of artifacts are of great importance for information systems (IS) research. DSR projects focus on specific problems (problem space), research the contexts, and elaborate solutions (solution space). The two most cited approaches that have emerged for conducting DSR projects are the three-cycle view of Hevner [13] and the DSR process by Peffers et al. [25]. Many researchers use these approaches to generate artifacts for specific problems [7]. Both approaches have in common that they enable the researcher to make a clear contribution to an application environment [13] and provide them with a roadmap for how to design artifacts in information systems [25].
In general, the "goal of DSR is to generate knowledge on how to effectively build innovative solutions to solve important problems effectively" [38, p. 5]. Thus, DSR projects make two key contributions while following the approaches of Hevner or Peffers and colleagues. First, they expand the solution space of suitable solutions to design novel and complex IT artifacts. Second, they generate valuable design knowledge that can be derived from designing artifacts. With an increasing number of DSR projects, the generation, accumulation, and codification of design knowledge are gaining in importance. Numerous scholars, such as vom Brocke et al. [38] and Rai [27], identify the need for approaches that effectively deal with the accumulation and codification of design knowledge in DSR in highcaliber IS journals [4].

Today, there are a plethora of ways to accumulate and codify design knowledge, such as design principles, design patterns, and design theories [38]. Design knowledge has certain characteristics that can change over time and space [17]. For example, design knowledge can be represented as tacit and explicit knowledge [26]. Tacit knowledge must first be externalized for transfer to other people. Tacit knowledge provides little information about concrete instructions for action and often contributes little to problem-solving. Thus, externalization transforms the knowledge into explicit knowledge, which can now be applied by others. Explicit design knowledge is easy to accumulate and codify [22]. In addition, knowledge owners are usually not aware of the distinctive knowledge characteristics and necessary steps to facilitate knowledge accumulation and utility. Without being aware of the characteristics of generated design knowledge and their properties, it remains difficult to make knowledge available in a useful form for others.

If we assess design knowledge as one of the most crucial aspects in DSR, a meaningful categorization 
and a shared understanding of their characteristics will be needed. In this regard, taxonomies can guide the classification of design knowledge representation to improve design knowledge accumulation and codification in DSR. However, IS research lacks a design knowledge taxonomy which would support the understanding of design knowledge properties. Thus, we present a taxonomy to facilitate the understanding of properties and representation of design knowledge. Therefore, we investigate the origin and distinctive characteristics of design knowledge, which is based on the following research question:

RQ: How can the representation and construction of design knowledge be represented in a taxonomy?

To answer our research question, we follow the methodology to develop taxonomies by Nickerson et al. [20], including the analysis of several design science research papers. We conduct a systematic literature review to identify meta-characteristics of the taxonomy. Afterwards, we evaluate the taxonomy with six domain experts and use two different cases to demonstrate the validity of our taxonomy by using two well-cited publications in the field of IS design science research and classify the design knowledge developed in the papers using the taxonomy. Our paper closes with a discussion of our results and suggestions for future research, limitations, and implications.

\section{Theoretical Background and Related Work}

Before diving deeper into the taxonomy development, we first want to show the relevance of designing artifacts for IS research. DSR has become an established and widely used research method in the field of IS research [7] for constructing artifacts [10] and is oftentimes structured through process methods $[13,25]$ to bring the practical development of artifacts into IS research.

In DSR in turn, design knowledge is becoming important for research and practice [11]. The DSR paradigm, in particular, focuses on the development and evaluation of technologies. Here, rules and concepts are applied, such as design theories and design principles, which can be used to map and support design processes [25]. DSR projects accumulate design knowledge through building, testing, and extending artifacts such as theory across different projects and publications [10]. The accumulation and codification of knowledge is a foundation stone for theories and enables professional practice [9]. Gregor et al. [11] remark on the meaning of how design knowledge is expressed as a theory to make design science formalizable.
Thus, we first take a closer look at what design knowledge is and how knowledge is generated. Simply put, a person is able to apply their knowledge and perform an action as soon as the person "knows" something [16]. The ability to create and use knowledge has become one of the most important characteristics [22]. There are different types of knowledge, i.e., tacit and explicit knowledge [21], which impact a person's ability to codify their personal knowledge [26]. Knowledge is created by an individual and becomes valuable by sharing it with other individuals [21]. While explicit knowledge can be easily transferred, other types of knowledge (such as tacit knowledge) are difficult to transfer [17].

Design knowledge is a special form of knowledge, namely knowledge to design a system including methods and constructs [10]. Designing (novel) artifacts and accumulating emerging design knowledge is an important component of DSR.

In IS research, there has long been an interest in analyzing design knowledge, understanding its components, and supporting its accumulation and codification. IS research around design knowledge is often based on the fundamentals of the knowledge creation theory by Nonaka [22]. Nonaka [21] emphasizes that the ability to create and use knowledge has become one of the most important human characteristics. Knowledge is created by an individual and becomes valuable by sharing it with other individuals [21].

In the early days of DSR, Walls et al. [40] already set the goals of a design theory in the description of both the properties of the artifact and the methods of construction to create the artifact. "Since design is both a noun and a verb, design is both a product and a process" [40, p. 42]. Thus, Walls et al. [40] use the term "meta-requirements" to describe the class of goals to which a design theory applies and abstract the design goal by addressing not the design of a specific design goal but a class of artifacts [40]. Van Aken defines design knowledge as " [...] knowledge that can be used to produce designs. The general design knowledge in the repertoire of the senior designer is compiled by him/ her over the years through formal education and through learning on the job" [37, p. 9].

Recent IS research, for example, by Chandra Kruse and Nickerson [4], has analyzed the essence of design in-depth and evaluated nine design elements that facilitate design knowledge accumulation. Vom Brocke et al. [38] propose a model that puts design knowledge into the context of problem space and solution space. Thus, the authors provide a framework on how to position design knowledge contribution in problem and solution space. 
Müller and Thoring [18] provide a typology of design knowledge consisting of four types of design knowledge, namely design artifacts, design intuition, design rationale, and design theories. Thus, they propose design knowledge can be represented in physical artifacts, as tacit gut feeling, as codified knowledge, or as scientific theories.

With our paper, we propose a holistic taxonomy that considers recent research dealing with design knowledge characteristics and properties. With the help of the taxonomy, design knowledge can be comprehensively analyzed to facilitate design knowledge accumulation and utility afterwards.

\section{Taxonomy Development}

We follow the methodology by Nickerson et al. [20] to achieve our goal of conceptualizing design knowledge to provide a taxonomy which supports researcher and practitioners in categorizing design knowledge. First, we conduct a literature review to achieve a comprehensive understanding of design knowledge. Second, we iteratively design our taxonomy. Third, we validate of our taxonomy using two application cases, thereby completing its systematic development.

We perform a systematic literature review according to Webster and Watson [41] and vom Brocke et al. [39] to derive meta-characteristics of design knowledge that are relevant for a design knowledge taxonomy. To cover a broad set of publications, we use the keywords "design knowledge" in the most prevalent databases to capture major journals and conferences in IS as well as related neighboring disciplines. The literature core is extended through forward and backward search [41].
We reduced the literature by eliminating doubles and papers that are out of our scope, such as papers that, for example, focus only on knowledge management and do not deal with design knowledge as a special form of knowledge. The focus in the paper should be either on conceptualizing design knowledge or properties of design knowledge. Thus, we excluded paper that focuses only on designing artifacts. In a second step, we analyzed the papers that were relevant for our analysis, as they fit our intention to develop a design knowledge taxonomy, resulting in the final number of 110 papers.

By developing a taxonomy, we pursue the goal of classifying different kinds of objects to improve understanding the (research) area [20]. We decided to use the most prominent and widely used approach in IS, i.e., Nickerson et al.'s [20] approach, because it offers the most systematic and step-by-step method for developing taxonomies.

In the first taxonomy step, we identify a metacharacteristic that will serve as a basis for our taxonomy development process. The identified metacharacteristic is the most comprehensive characteristic, and the added characteristics will be a logical consequence of this meta-characteristic. For the selection of meta-characteristics, we use our goal to conceptualize expressions of design knowledge and thus facilitate design knowledge accumulation.

The advantage of Nickerson et al.'s method [20] is that it is iterative and the search for additional characteristics and dimensions is performed until the taxonomy is complete. Thus, we need ending conditions (EC) that help us find the time for the completion of the taxonomy development. We define our ending conditions as the following:

\begin{tabular}{|c|c|c|c|c|c|}
\hline & $>$ Iteration & Iteration 2 & Iteration 3 & Iteration 4 & Iteration 5 \\
\hline \multirow[t]{5}{*}{ Approach } & $\begin{array}{l}\text { Empirical to } \\
\text { conceptual }\end{array}$ & $\begin{array}{l}\text { Conceptual to } \\
\text { empirical }\end{array}$ & $\begin{array}{l}\text { Empirical to } \\
\text { conceptual }\end{array}$ & $\begin{array}{l}\text { Conceptual to } \\
\text { empirical }\end{array}$ & $\begin{array}{l}\text { Conceptual to } \\
\text { empirical }\end{array}$ \\
\hline & Occurrence & Occurrence & Occurrence & Occurrence & Occurrence \\
\hline & Aim of design & Aim of design & Aim of design & Aim of design & Aim of design \\
\hline & Expression & Expression & Expression & Expression & Expression \\
\hline & & Main formulation & Main formulation & Main formulation & Main formulation \\
\hline \multirow[t]{5}{*}{ Dimensions } & & & Codification & & \\
\hline & & & Unit of design & Unit of design & Unit of design \\
\hline & & & Maturity & Maturity & Maturity \\
\hline & & & & Primary derivation & Primary derivation \\
\hline & & & & & Level of abstraction \\
\hline $\begin{array}{l}\text { Ending } \\
\text { Conditions }\end{array}$ & & $(2)$ & (2) & (1), (2) & (1), (2), (3), (4) \\
\hline Sum & 3 & 4 & 7 & 8 & 9 \\
\hline
\end{tabular}

Fig. 1 Iteration and Dimensions 
(1) All identified papers have been examined,

(2) at least one object must be classified under every characteristic of every dimension,

(3) no dimensions or characteristics can be added to the final iteration, dimensions, and characteristics, and

(4) cell combinations are unique and should not repeat.

Now that the ending conditions are defined, the iterative taxonomy development process can start, beginning with either an empirical-to-conceptual, a conceptual-to-empirical approach, or a combination of both to identify additional characteristics and dimensions (see Figure 1). A conceptual-to-empirical approach involves the examination of empirical cases to see how they fit within the conceptualization, while an empirical-to-conceptual approach involves starting with empirical data clusters before conceptualizing the nature of the elements [20]. Our taxonomy development starting point is based on the work of Gregor and Hevner's analyses of theories [9], Nonaka's knowledge creation theory [22], and vom Brocke et al.'s journey of design knowledge in DSR [38]. Thus, we start with an empirical-to-conceptual approach. As soon as the ending conditions are met, the taxonomy development process is terminated, followed by evaluating the developed taxonomy.

Nickerson et al. [20] advise for each dimension features that are mutually exclusive and collectively exhaustive. Since this is clearly not possible in each dimension due to the complexity of design knowledge and its properties, we have chosen to follow the wellestablished approaches of [8] and [31] and allow the assignment of an element to multiple characteristics of a single dimension. Thus, we remove their mutual exclusivity [20], including the column exclusive that shows whether a characteristic is exclusive (Y) or nonexclusive $(\mathrm{N})$.

Assessing the usefulness of taxonomies is a critical evaluation step [35]. One usefulness evaluation approach that Nickerson et al. [20] propose is the evaluation with users. Therefore, we conduct semistructured expert interviews ( $\mathrm{N}=6,2$ females, 4 males). Four of the experts are from the research community and have either relevant publication in the field of DSR or expertise in developing and publishing taxonomies. The interviewed practitioner has two years of practical experience in designing and developing IT systems.

We use the interviews to revise our taxonomy and determine its usefulness in developing and understanding design knowledge among practitioners and researchers. The interviews lasted between 30 and 45 minutes and were conducted using an interview guideline consisted of open questions regarding the five evaluation criteria whether the taxonomy is concise, robust, comprehensive, extendible, and explanatory. The main point of criticism was that formulation of the dimensions and characteristics are ambiguous such as the naming of the dimension "Expression" which was understood differently by the interviewees. Oftentimes, the descriptions of the dimensions and characteristics were not clear and were therefore adjusted accordingly. Some statements also contained suggestions for possible applications of the taxonomy and how it can be integrated into IS research. In particular, the extent to which design knowledge should be at the core of the taxonomy and the level at which it should be integrated were considered. Many DSR papers use design principles, for example, but these vary widely in their formulation and implementation. In the end, it was decided not to be very specific here and to choose the dimension occurrence and add the dimension codification, which was previously partially integrated in the dimension expression, but did not provide sufficient delimitation here. Thus, the interviews provide valuable approaches for the revision of the taxonomy.

\section{A Taxonomy of Design Knowledge}

In the following, we present our final taxonomy. The taxonomy consists of the three metacharacteristics knowledge generation, knowledge purpose, and knowledge representation that all refer to design knowledge. Each meta-characteristic can be divided into two or three dimensions. According to $[8$, 29], we visualize the taxonomy as a morphological box to illustrate the relationships between the dimensions and characteristics (see Figure 2). After presenting the taxonomy here in general, we will apply it to two cases in section 5 and present exemplary characteristics.

\subsection{Meta-Characteristic Knowledge Generation}

The first meta-characteristic knowledge generation refers to the source of design knowledge. Thus, design knowledge can be created in a variety of ways, which is an important characteristic to understand its nature. The dimension knowledge generation can be divided into the three dimensions occurrence, primary derivation, and aim of design. The dimension occurrence sums up, how the design knowledge is generated. Thus, knowledge may be generated through principles of form and function [9], through instantiated implementation [9], through a prototypical design [15], through the development of a method [25], or by developing models [14, 28]. While principles of form and function try to describe 


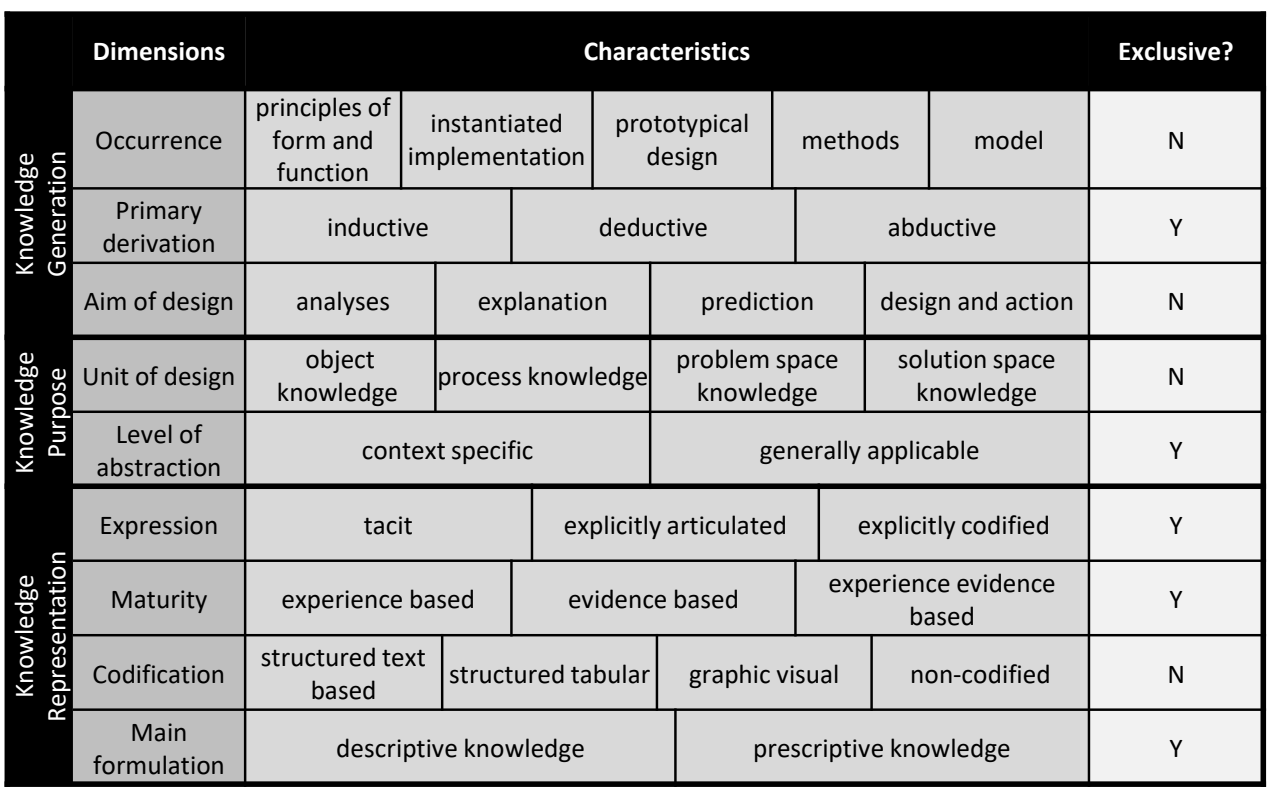

Fig. 2 Taxonomy of Design Knowledge

the design of artifacts generally and provide instructions on how to design elements, a prototypical design or implementation aims at designing concrete systems. These are often accompanied by visual representations of the design elements. The origin of design knowledge can also be of a formal nature and develop through modeling. Methods, on the other hand, often provide step-by-step instructions and give the user concrete directions to do something.

Depending on how design knowledge is derived, different approaches can be distinguished between. These are important for the classification and the relation of the knowledge and represent the dimension primary derivation. This dimension can be further divided into inductive, deductive, and abductive and allows conclusions about the creation process. Design knowledge based on empiricism is called inductive,

while the basis on theories can be called deductive. Abduction, on the other hand, is based on the invocation of an explanatory hypothesis.

Normally, generating design knowledge goes hand in hand with an underlying intention. Design knowledge is not only created in different ways but also for different intentions, which we map in our taxonomy under the dimension aim. Thus, we refer to Gregor et al.'s general notion of theory in IS [9] and break down the design knowledge aim into the four characteristics analysis, explanation, prediction, and design and action. By observing and analyzing a fact or an artifact, insights can be drawn that can be used to expand the body of knowledge. For example, case studies [42] analyze a specific situation and generate valuable design knowledge for future purposes, whereas the characteristic explanation produces design knowledge of explanatory nature, and thus the knowledge explains causal relationships. The characteristic prediction tells what will happen in the future if certain conditions are met [11]. The last characteristic design and action provides someone with concrete design information on how to do something. Thus, the knowledge makes explicit prescriptions on how to design an artifact [11].

\subsection{Meta-Characteristic Knowledge Purpose}

The meta-characteristic knowledge purpose includes all characteristics around the individual components of design knowledge. Thus, the goal is to analyze the components that comprise design knowledge. The meta-characteristic is further split into two dimensions, namely unit of design and level of abstraction. While unit of design considers individual elements of knowledge, level of abstraction refers to the abstraction and applicability of knowledge to other (new) contexts.

Regarding the unit of analysis dimension, object knowledge provides knowledge on the properties about an artifact. Thus, the knowledge about an area is a crucial feature of design knowledge. To develop an artifact, you need knowledge about the characteristics and properties of the artifact and its materials [37]. Walls et al. [40] describe object knowledge with "meta-design", which is not meant to be for a specific artifact but a class of artifacts.

Furthermore, process knowledge, the knowledge of how to get to the solution, is often a characteristic 
for reusability [4, 23]. Process knowledge is about the characteristics and properties of design processes, which can be used to produce designs [37]. Process knowledge provides explicit prescriptions for constructing an artifact by solving a problem [12].

The last distinction in the dimension components focuses on design knowledge differs in terms of goals and scope, which allows for conclusions as to whether knowledge belongs to solution or problem spaces [1, 33, 38]. Information dealing with problem context, and key stakeholders in problem spaces can be summarized as problem space knowledge. Usually, the knowledge is mostly descriptive knowledge, describing current and desired situations [30]. Understanding the underlying problem is crucial for choosing design solutions.

Knowledge in solution space can be generated through creating, assessing, and refining DSR results [38]. Solutions for arising problems represent the core of shared design knowledge. Thus, design knowledge consists of elements of the solution space, such as knowledge about how an artifact should be designed as well as whether and why particular designs are desirable [24]. Solutions change, adapt, and often improve in the process of new application contexts. In addition, knowledge is dynamic and continually revised [22].

The second dimension, level of abstraction refers to the generalization of design knowledge and represents the design knowledge projectability. A solution is generally applicable if it is abstract, so it shows solutions that fit in different projects. Through the recurring application of a solution, it is evaluated and revised if necessary. Depending on the degree of abstraction, the knowledge is either context specific and usually cannot be transferred to new application areas, or it is generally applicable. Context specific knowledge often includes design knowledge about very specific artifacts whose knowledge has not really been abstracted and is therefore not universally applicable. General applicable design knowledge, on the other hand, is usually abstracted in such a way that it can be applied to different artifact classes and objects independently of the design context.

\subsection{Meta-Characteristic Knowledge Representation}

Our taxonomy ends with the meta-characteristic knowledge representation, which classifies the design knowledge according to its form of representation. Knowledge representation is further subdivided into the four dimensions expression, maturity, codification, and main formulation. The ability to create and use knowledge has become one of the most important characteristics [22]. There are two types of knowledge, i.e., tacit and explicit knowledge [21], which impact a person's ability to codify their personal knowledge [26]. Knowledge is generated by an individual and becomes valuable by sharing it with other individuals. While explicit knowledge can be easily transferred, tacit knowledge is difficult to transfer [17]. Explicit knowledge can be codified either textually, digitally, or on paper and thus passed on (explicit codified). Another way of representing explicit knowledge is auditory transmission when the knowledge is explicitly articulated.

The maturity dimension looks at the extent to which design knowledge has already proven itself experimentally or practically, whereas evidence based design knowledge is based on empirical evidence and has usually proven itself theoretically. Design knowledge that has been used both experimentally and evidence based is categorized as experience evidence based.

The codification of design knowledge can be seen as the creation of explicit knowledge from the solution space [2]. Mostly, prescriptive knowledge is about the (ideal) target state. In addition to the target state, rules and specifications for the correct implementation of a solution can be included. The solution knowledge should provide the user with an understanding of the consequences of their decisions. There are many approaches to codifying design knowledge in IS and other research and practice. As a rule, these differ in their presentation. Structured text-based codification approaches focus on codification in texts. Other approaches codify design knowledge in tabular form to present the information clearly. When it comes to interface design, for example, graphic visual codification approaches are used to convey the knowledge. Design knowledge that has not yet been externalized is often available as non-codified knowledge. A mixture of all approaches is also possible (mixed). Thus, the dimension is not exclusive, and a combination of various characteristics is possible.

The dimension formulation distinguishes between descriptive and prescriptive. Thus, descriptive knowledge describes knowledge that can be expressed in a declarative sentence or indicative proposition [36], while prescriptive knowledge provides explicit prescriptions (e.g., methods, techniques, principles of form and function) for designing an artifact $[3,11]$.

\section{Application of the Taxonomy}

We demonstrate and evaluate our taxonomy by applying it to two contrasting design science research papers (see Table 1). Thus, we conduct two case applications to demonstrate how the taxonomy supports the analysis of design knowledge properties. 


\subsection{Application Case 1}

The first application case is a paper by Nguyen et al. [19] which is a very recent DSR paper published in the European Journal of Information Systems (EJIS) in the year 2020. The goal of the paper is to develop "design principles for learning analytics information systems to establish a foundation for further development and implementation of learning analytics to support learning and teaching in higher education" [19, p. 1]. Therefore, this paper is well suited to classify the generated design knowledge.

We start analyzing the paper with the first metacharacteristic design knowledge generation to analyze the origin of the design knowledge in the paper. The paper develops design principles whose practical implementation is presented and evaluated in a prototypical design. Thus, we classify the dimension occurrence in our characteristic prototypical design. For the prototypical design, design principles are first derived. The primary derivation, our second dimension, thus, is deductive, based on learning analytics literature, learning analytics processes literature, and kernel theories. Afterwards, the paper derives inductive empirical conclusions. Nevertheless, this dimension focuses on the initial origin of design knowledge. Further aspects are classified in the dimension maturity.

\begin{tabular}{|c|c|c|}
\hline Dimension & $\begin{array}{c}\text { Characteristics } \\
\text { Application } \\
\text { Case } 1 \text { [19] }\end{array}$ & $\begin{array}{c}\text { Characteristics } \\
\text { Application } \\
\text { Case } 2[34]\end{array}$ \\
\hline Occurrence & $\begin{array}{c}\text { Prototypical } \\
\text { design }\end{array}$ & Methods \\
\hline $\begin{array}{c}\text { Primary } \\
\text { Derivation }\end{array}$ & Deductive & Deductive \\
\hline $\begin{array}{l}\text { Aim of } \\
\text { Design }\end{array}$ & Design and action & $\begin{array}{c}\text { Analyses, } \\
\text { explanation }\end{array}$ \\
\hline $\begin{array}{l}\text { Unit of } \\
\text { Design }\end{array}$ & $\begin{array}{c}\text { Object } \\
\text { knowledge, } \\
\text { process } \\
\text { knowledge, } \\
\text { problem-space } \\
\text { knowledge, } \\
\text { solution-space } \\
\text { knowledge }\end{array}$ & $\begin{array}{c}\text { Object knowledge, } \\
\text { process } \\
\text { knowledge, } \\
\text { problem-space } \\
\text { knowledge, } \\
\text { solution-space } \\
\text { knowledge }\end{array}$ \\
\hline $\begin{array}{c}\text { Level of } \\
\text { Abstraction }\end{array}$ & Context-specific & $\begin{array}{l}\text { Generally } \\
\text { applicable }\end{array}$ \\
\hline Expression & Explicit codified & Explicit codified \\
\hline Maturity & Experience-based & Experience-based \\
\hline Codification & $\begin{array}{l}\text { Structured text- } \\
\text { based, graphic- } \\
\text { visual }\end{array}$ & $\begin{array}{l}\text { Structured text- } \\
\text { based }\end{array}$ \\
\hline $\begin{array}{c}\text { Main } \\
\text { Formulation }\end{array}$ & $\begin{array}{c}\text { Prescriptive } \\
\text { knowledge }\end{array}$ & $\begin{array}{l}\text { Prescriptive } \\
\text { knowledge }\end{array}$ \\
\hline
\end{tabular}

Tab. 1 Application of the Taxonomy on Two Cases
The second meta-characteristic knowledge purpose focuses on properties of design knowledge. Thus, the dimension unit of design classifies the composition of the design knowledge. The authors provide design knowledge regarding learning analytics information systems, so object knowledge that supports the reader to understand the underlying design object and related problems. By providing design principles and design solutions, they analyze the problem space and provide design solutions from the solution space. Therefore, we classify the characteristics object knowledge, process knowledge, problem-space knowledge, and solution-space knowledge in the dimension unit of design. The dimension unit of design is not exclusive, which allows us to select several elements. The generated design knowledge is related to learning analytics research. Thus, the authors generate context specific design knowledge.

Finally, we consider the meta-characteristic knowledge representation to analyze how the design knowledge is represented. The design knowledge expression is explicitly codified through design principles. The developed design principles are evaluated in a proof-of-concept evaluation. Thus, the dimension maturity classifies the design knowledge in the characteristic experience based. Regarding the dimension codification, we categorize the design principles as structured text based and the prototypical design as graphic-visual. Overall, the authors of the paper use prescriptive design knowledge with the goal of providing a foundation of learning analytics for developers and researchers.

\subsection{Application Case 2}

The second paper by Siponen et al. [34] is in contrast to our first application case less recent but well cited and develops a design theory for secure information systems design methods. Compared to case 1, the paper is somewhat older, namely from 2006 and published in the Journal of the Association for Information Systems (JAIS). The goal of the paper is to develop a secure information system design theory framework. First, the authors derive design requirements for secure information system design methods and show that known design methods fail to satisfy these requirements. Second, the paper describes and demonstrates a design method that addresses these requirements.

Thus, regarding the dimension occurrence we choose the characteristic methods because the authors' goal is developing a design theory for secure information systems. The primary design knowledge derivation is based on kernel theories and is thus deductive. The authors pursue the intention to design 
requirements and to analyze recent papers that fail to meet the requirements. Thus, we choose the element analysis, explanation and design and action because, in addition to the analysis, they also show how it can be done better and develop a framework for this. Within the second meta-characteristic, we consider the properties of the design knowledge. The dimension unit of design allows us to draw conclusions about the individual design knowledge elements. In this paper, they first describe the object of design, namely secure information systems and their special features. Process knowledge is represented in a process model. The meta-requirements tackle the problem space and provide solutions from the solution space. Thus, we choose the characteristics object knowledge, process knowledge, problem-space knowledge, and solutionspace knowledge. Regarding the level of abstraction and the main idea of design theories, we classify the design knowledge as generally applicable.

The third meta-characteristic knowledge representation analyzes the design knowledge presentation. The authors provide explicit codified design knowledge that is text-based structured. The meta-requirements and the design theory offer prescriptive design knowledge on how to design secure information systems. In conclusion, the authors evaluate the design theory framework through two empirical studies that demonstrate the validity of the proposed framework [34]. Thus, the design knowledge is experience based.

\section{Discussion, Limitations, and Future Work}

The goal of our paper was to develop a design knowledge taxonomy and, thus, to contribute to a deeper understanding of design knowledge properties. Therewith, we pursued the RQ on how the representation and construction of design knowledge can be represented in a taxonomy. To answer our RQ, we follow the methodology of Nickerson et al. [20]. Our taxonomy is based on a systematic literature review to ground our taxonomy on previous literature. Followed by this, we followed a combination of empirical-to-conceptual and conceptual-to-empirical approaches. Afterwards, we evaluate the taxonomy by interviewing six domain experts and demonstrate its practical application and utility.

With the taxonomy, we contribute to a better scientific understanding of design knowledge properties by providing an instrument to further understand, design, and accumulate design knowledge. A large amount of design knowledge is generated in research and practice. In some contexts, this is consciously developed, perceived, and codified; in other contexts, however, design knowledge is generated incidentally, and its dissemination does not play a decisive role. Design knowledge has certain characteristics that make it difficult to share, especially if it is not represented in a codified form [17]. The lack of reuse also brings with it that the generated design knowledge does not leap from research into practice. The IS research community observes that DSR projects generate a large amount of design knowledge, but the knowledge often ends as a single success story [4]. Thus, the design knowledge is often lost at the end of the projects. The limited design knowledge accumulation in the IS community is problematic, as single contributions tend to remain isolated with little to no relation to other solutions [38]. This is accompanied by the problem that valuable knowledge is lost, although it could be useful in new projects. Our taxonomy application demonstrates that the accumulation of design knowledge can look very different. While some studies focus on textual and visual accumulations, others focus on only textual ways $[12,40]$. Design patterns as an example to codify proven design solutions may be advantageous in terms of reusability compared to other approaches [6]. The taxonomy has proven to be helpful in application to structure design knowledge. The categories allow us to identify which information is missing in sharing ideas and solutions. Since to solve a problem, you must first understand the problem, understand where the solution can go, understand the artifact and the situation, and then use the process knowledge to find a way to achieve the solution. If one piece of these information is missing, the added value of the accumulated design knowledge decreases with increasing time and thus also the reusability.

In IS and practice, there are various approaches that accumulate and codify design knowledge, such as design principles, design patterns, and design theories [38]. We see two important points in making design knowledge more usable and accessible so that it can be shared and reused in new application contexts. First, the tool in which the design knowledge is codified must enable the user to find the necessary (design) information in the shortest possible time. Second, the way the design knowledge is codified is crucial for the success of the tool. With the taxonomy we want to support the latter by providing a foundation for analyzing and understanding the characteristics and properties of design knowledge. Therefore, we would like to show with our contribution that design knowledge, if properly prepared for codification, does not have to end as a single success story. DSR projects should not focus only on achieving the (design) solution but should consider sustainability and reusability in their contributions. 
These initial findings could be used in future work that could, for example, develop a framework for processing and accumulating design knowledge. Based on the taxonomy and practical implementations, the framework as a design tool could derive tips and guidance for (IS) researchers and practitioners to use their design knowledge to facilitate design knowledge accumulation and utility. In addition, future work can classify existing design knowledge using the taxonomy, as we did in section 5 with two example papers, and use it to draw conclusions about the reusability [4] and projectability [38] of design knowledge.

Our study is limited by a few factors that provide directions for future research. Based on the literature review and the evaluation with six experts, we firmly establish the wording, the scope, and the completeness of the taxonomy. Nevertheless, the developed taxonomy is a time-bound snapshot that needs to be updated frequently to remain relevant and to consider new dimensions and characteristics in the future. As also addressed by Nickerson et al. [19], it must remain guaranteed that the taxonomy can be extended in the future without difficulty. We have also taken this into account in our interview guide.

We based the naming of meta-characteristics, dimensions, and characteristics as best as possible on the common understanding of the related literature to achieve familiar terms and as few comprehension problems as possible. Likewise, with the help of section 4 and the application of the taxonomy in section 5, we put the taxonomy into context and to classify practical design knowledge with the help of the taxonomy.

\section{Conclusion}

The goal of our paper was the analysis of design knowledge properties to develop a design knowledge taxonomy and to conceptualize design knowledge properties. Thus, we follow the taxonomy development methodology by Nickerson et al. [20], evaluate the taxonomy in expert interviews, and demonstrate the taxonomy application through two cases.

From our results, we can draw several implications for theory and practice. Regarding scientific contributions, our work contributes to a deeper understanding of the design knowledge accumulation field. Many IS and especially DSR researchers are engaged in supporting researchers and practitioners in the accumulation and codification of design knowledge [5, 27, 30, 32, 38]. We aim to contribute to this with the aid of our taxonomy and to provide a tool that supports the analysis and classification of design knowledge properties. Furthermore, the taxonomy can be the starting point for frameworks that facilitate design knowledge accumulation and utility. Before design knowledge can be codified and passed on according to specific rules, knowledge owners must first be aware of the properties and particularities of design knowledge.

\section{Acknowledgements}

The second author acknowledges funding from the Basic Research Fund (GFF) of the University of St. Gallen.

\section{References}

[1] Baskerville, R., A. Baiyere, S. Gergor, A. Hevner, and M. Rossi, "Design Science Research Contributions: Finding a Balance between Artifact and Theory", JAIS, 19(5), 2018, pp. 358-376.

[2] Baskerville, R. and J. Pries-Heje, "Design Theory Projectability", 2014, pp. 219-232.

[3] Chandra, L., S. Seidel, and S. Gregor, "Prescriptive Knowledge in IS Research: Conceptualizing Design Principles in Terms of Materiality, Action, and Boundary Conditions", HICSS, 2014, pp. 4039-4048.

[4] Chandra Kruse, L. and J.V. Nickerson, "Portraying Design Essence", HICSS, 2018, pp. 4433-4442.

[5] Chandra Kruse, L., S. Seidel, and S. Purao, "Making Use of Design Principles", DESRIST, 2016, pp. 37-51.

[6] Dickhaut, E., A. Janson, and J.M. Leimeister, "Codifying Interdisciplinary Design Knowledge through Patterns - The Case of Smart Personal Assistants", DESRIST, 2020.

[7] Engel, C., N. Leicht, and P. Ebel, "The Imprint of Design Science in Information Systems Research: An Empirical Analysis of the AIS Senior Scholars' Basket", ICIS 2019 Proceedings, 2019.

[8] Gelhaar, J., T. Groß, and B. Otto, "A Taxonomy for Data Ecosystems", Hawaii International Conference on System Sciences 2021, 2021.

[9] Gregor, S., "The nature of theory in information systems", MIS Quarterly 30 (3), 2006.

[10] Gregor, S. and A.R. Hevner, "Positioning and presenting design science research for maximum impact", MIS Quarterly, 2013, pp. 337-355.

[11] Gregor, S. and D. Jones, "The Anatomy of a Design Theory", Association for Information Systems, 8(5), 2007.

[12] Gregory, R.W. and J. Muntermann, "Heuristic Theorizing: Proactively Generating Design Theories", Information Systems Research, 25(3), 2014, pp. 639-653.

[13] Hevner, A.R., "A three cycle view of design science research", Scandinavian journal of information systems(19(2)), 2007.

[14] Li, M.M., C. Peters, and J.M. Leimeister, "A Hypergraph-Based Modeling Approach for Service 
Systems", in Advances in Service Science: Proceedings of the 2018 INFORMS International Conference on Service Science, H. Yang and R. Qiu, Editors, Cham, 2019. 2019. Springer International Publishing: Cham.

[15] Lim, Y.-k., E. Stolterman, and J. Tenenberg, "The anatomy of prototypes", ACM Transactions on ComputerHuman Interaction, 15(2), 2008, pp. 1-27.

[16] McLure-Wasko, M., "How are Knowledge Management Systems Different from Information Systems, and Who Cares?", AMCIS, 1999, pp. 486-488.

[17] Müller, R.M. and K. Thoring, "A Typology of Design Knowledge: A Theoretical Framework", Americas Conference on Information Systems (AMCIS) 2010 Proceedings, 2010, pp. 300-310.

[18] Müller, R.M. and K. Thoring, "A Typology of Design Knowledge: A Theoretical Framework", Americas Conference on Information Systems (AMCIS) Proceedings, 2010.

[19] Nguyen, A., T. Tuunanen, L. Gardner, and D. Sheridan, "Design principles for learning analytics information systems in higher education", European Journal of Information Systems, 2020, pp. 1-28.

[20] Nickerson, R.C., U. Varshney, and J. Muntermann, "A method for taxonomy development and its application in information systems", European Journal of Information Systems, 22(3), 2013, pp. 336-359.

[21] Nonaka, I. and H. Takeuchi, The Knowledge-Creating Company: How Japanese Companies Create the Dynamics of Innovation, Oxford University Press, 1995.

[22] Nonaka, I. and R. Toyama, "The knowledge-creating theory revisited: knowledge creation as a synthesizing process", Knowledge Management Research \& Practice, 1(1), 2003, pp. 2-10.

[23] Offermann, P., S. Blom, and U. Bub, "Strategies for creating, generalising and transferring design science knowledge-a methodological discussion and case analysis", WI, 2011.

[24] Österle, H., J. Becker, U. Frank, T. Hess, D. Karagiannis, H. Krcmar, P. Loos, P. Mertens, A. Oberweis, and E.J. Sinz, "Memorandum on design-oriented information systems research", European Journal of Information Systems, 20(1), 2011, pp. 7-10.

[25] Peffers, K., T. Tuunanen, M.A. Rothenberger, and S. Chatterjee, "A Design Science Research Methodology for Information Systems Research", JMIS, 2007, pp. 45-77.

[26] Polanyi, M., "Tacit Knowing: Its Bearing on Some Problems of Philosophy", Reviews of modern physics, 34(4), 1962, pp. 601-616.

[27] Rai, A., "Celebrating 40 Years of MIS Quarterly: MISQ's History and Future Through the Lenses of its Editors-in-Chief", MIS Quarterly, 40, 2016.

[28] Recker, J., R. Lukyanenko, M. Jabbari, B.M. Samuel, and A. Castellanos, "From Representation to Mediation: A
New Agenda for Conceptual Modeling Research in a Digital World", MIS Quarterly, 45(1), 2021, pp. 269-300.

[29] Ritchey, T., "Problem structuring using computer-aided morphological analysis", Journal of the Operational Research Society, 57(7), 2006, pp. 792-801.

[30] Rothe, H., L. Wessel, and A.P. Barquet, "Accumulating Design Knowledge: A Mechanisms-Based Approach", Journal of the Association for Information Systems, 21(3), 2020, pp. 771-810.

[31] Schöbel, S.M., A. Janson, and M. Söllner, "Capturing the complexity of gamification elements: a holistic approach for analysing existing and deriving novel gamification designs", European Journal of Information Systems (EJIS), 2020.

[32] Seidel, S., L. Chandra Kruse, N. Székely, M. Gau, and D. Stieger, "Design principles for sensemaking support systems in environmental sustainability transformations", European Journal of Information Systems, 27(2), 2018, pp. 221-247.

[33] Simon, H.A. and J.E. Laird, The sciences of the artificial, 2019th edn., The MIT Press, Cambridge Massachusetts, London England, 1996.

[34] Siponen, M., R. Baskerville, and J. Heikka, "A Design Theory for Secure Information Systems Design Methods", 1536-9323, 7(11), 2006, pp. 725-770.

[35] Szopinski, D., T. Schoormann, and D. Kundisch, "Because your Taxonomy is Worth it: Towards a Framework for Taxonomy Evaluation", ECIS, 2019.

[36] Tokuhama-Espinosa, T., Mind, brain, and education science: A comprehensive guide to the new brain-based teaching, W.W. Norton, New York, NY, 2011.

[37] van Aken, J.E., "Valid knowledge for the professional design of large and complex design processes", Design Studies, 26(4), 2005, pp. 379-404.

[38] vom Brocke, J., R. Winter, A. Hevner, and A. Maedche, "Accumulation and evolution of design knowledge in design science research - A journey through time and space", Journal of the Association for Information Systems (JAIS), 23(3), 2020, pp. 9-49.

[39] Vom Brocke, J., A. Simons, K. Riemer, B. Niehaves, R. Plattfault, and A. Cleven, "Standing on the Shoulders of Giants: Challenges and Recommendations of Literature Search in Information Systems Research", Communications of the Association for Information Systems (CAIS), 2015.

[40] Walls, J.G., G.R. Widmeyer, and O.A. El Sawy, "Building an Information System Design Theory for Vigilant EIS", Information Systems Research, 3(1), 1992, pp. 36-59.

[41] Webster, J. and R.T. Watson, "Analyzing the Past to Prepare for the Future: Writing a Literature Review", MIS Quarterly, 2002, pp. 13-23.

[42] Yin, R.K., Case study research and applications: Design and methods, SAGE, Los Angeles, 2018. 